\title{
Changing the future: An initial test of Future Specificity Training (FeST)
}

\author{
D.J. Hallford ${ }^{a, *}$, J.J.E. Yeow ${ }^{\mathrm{a}}$, G. Fountas ${ }^{\mathrm{a}}$, C.A. Herrick ${ }^{\mathrm{a}}$, F. Raes ${ }^{\mathrm{b}}$, A. D'Argembeau ${ }^{\mathrm{c}}$ \\ ${ }^{a}$ School of Psychology, Deakin University, 221 Burwood Hwy, Burwood, Victoria, 3125, Melbourne, Australia \\ ${ }^{\mathrm{b}}$ Faculty of Psychology and Educational Sciences, KU Leuven, Tiensestraat 102, Box 3712, 3000, Leuven, Belgium \\ ${ }^{\mathrm{c}}$ Psychology and Neuroscience of Cognition Research Unit, Department of Psychology, University of Liège, Belgium and Fonds de La Recherche Scientifique (F.R.S.-FNRS), \\ Belgium
}

\section{A R T I C L E I N F O}

\section{Keywords:}

Episodic future thinking

Future thinking

Specificity

Mental imagery

Anticipatory pleasure

Perceived control

\begin{abstract}
A B S T R A C T
A range of psychiatric disorders are characterised by impairments in episodic future thinking (EFT), and particularly simulating specific, spatiotemporally-located future events. No study has examined whether training can lead to sustained improvement in specific EFT. In this study, participants $(N=60 ; M$ age $=31, S D=13.2)$ were randomized to a two-session, group-based future thinking program (Future Specificity Training; FeST) or wait-list. At follow-up the training group, relative to wait-list, showed large, statistically-significant improvements in the ability to mentally simulate specific EFT $(d=.82)$, increases in detail $(d=1.32)$, use of mental imagery $(d=1.32)$, anticipated $(d=1.78)$ and anticipatory pleasure $(d=1.07)$, perceived control $(d=1.20)$, and likelihood of occurrence $(d=1.09)$. Some effects were also observed on positive, generalised future selfstates. In the context of inherent limitations of subjective reporting in trials, this study provides evidence that EFT specificity can be enhanced, and the effects of FeST indicate a possible avenue to disrupt psychopathological processes.
\end{abstract}

\section{Introduction}

Thinking about events that might occur to one's self in the future, referred to as episodic future thinking (EFT; Atance \& O'Neill, 2001 , $\mathrm{Dec}$ ), is a crucial mental process in healthy functioning. Specificity in EFT refers to simulating particular events that are located in time and space, relative to abstracted ideas of one's future, or categories of events that could occur. For example, imagining an upcoming dinner with a particular friend at a chosen restaurant would constitute a specific EFT, whereas the abstracted, general thought of having good relationships with friends in the future would not. Specific EFT, therefore, involves simulating contextual details, such as a spatial environment, the day or time of day, sequences of events, sensory details, and thoughts or emotions that one might experience. Such details tend to increase the sense of pre-experiencing the future (D'Argembeau, Lardi, \& Van der Linden, 2012), and the ease of simulation increases the perceived likelihood of imagined events (Kahneman \& Tversky, 1981).

These characteristics of EFT are implicated in a range of functions in healthy adaptation, such as planning, goal-directed behaviour, emotional regulation, and decision-making (Schacter, Benoit, \& Szpunar, 2017). For example, studies show that increased detail in EFT is associated with more perceived control over future events (e.g., Boland, Riggs, \& Anderson, 2018; Jing, Madore, \& Schacter, 2016) and improved emotion regulation (Jing et al., 2016, 2019). Detail and imagery for specific EFT have been linked with stronger anticipated pleasure (the prediction of how pleasurable a future event would be) and anticipatory pleasure (how pleasurable it is to think about a future event) for positive events (Boland et al., 2018; Hallford et al., 2020). Moreover, engaging in future thinking can have effects on decisionmaking on whether to choose larger, longer-term rewards over immediate, smaller rewards, which is particularly relevant for health-related behaviours (e.g., Daniel, Stanton, \& Epstein, 2013b, 2013a; Snider, LaConte, \& Bickel, 2016; Stein, Tegge, Turner, \& Bickel, 2018), and impulsivity in general.

Given the role of EFT in healthy functioning, it is notable that it is impaired in a range of psychiatric disorders, with reduced specificity, detail and imagery in disorders such as clinical depression, schizophrenia-spectrum, and bipolar disorder (Hallford, Austin, Takano, \& Raes, 2018; Holmes, Blackwell, Burnett Heyes, Renner, \& Raes, 2016). These deficits may maintain pathological processes. For example, impaired detail and imagery for positive future events predict lower anticipatory pleasure in major depressive disorder (Hallford et al., 2020), which is linked with motivation (Engel, Fritzsche, \& Lincoln, 2013; Sherdell, Waugh, \& Gotlib, 2012) and psychosocial functioning (Foussias et al., 2011). Anhedonia can be a difficult to treat symptom of depression, and is a marker of the course of illness and treatment

\footnotetext{
* Corresponding author.

E-mail address: david.hallford@deakin.edu.au (D.J. Hallford).
} 
responses (Dunn \& Roberts, 2016). Enhancing positive affect when anticipating future events may be one method of combatting anhedonia (see Schubert, Aloo, Scharfen, \& Morina, 2019 for a review of change in positive affect after future simulations). Other mechanisms linking future thinking to psychopathology include lower detail related to hopelessness (MacLeod et al., 2005) and difficulty in simulating future events being associated with apathy (Raffard, Esposito, Boulenger, \& Van der Linden, 2013). Training interventions to improve specificity in future thinking might be useful in redressing these types of deficits. They may also have other application in enhancing EFT functioning or outcomes in healthy populations too. For example, Brown, Macleod, Tata, and Goddard (2002) found that women who were pregnant for the first time were less worried and more able to cope with events leading up to the birth when they could more realistically simulate these experiences. Similarly, Taylor, Pham, Rivkin, and Armor (1998) reported that students who mentally simulated studying using specific EFT reported subsequent increases in hours of study and improved exam performance.

To the authors' knowledge, to date no study has examined a training program for specific EFT. Instead, research into improving specificity in episodic thinking has primarily focused on memory, with Memory Specificity Training (MeST) showing success in training healthy and psychiatric populations to increase their ability to retrieve specific memories (see (Barry, Sze, \& Raes, 2019) for a review). Although future thinking relies on memory to construct mental simulations (Schacter, Addis, \& Buckner, 2007), the outcomes of MeST on future thinking are currently unknown. Programs such Future-Directed Therapy (Vilhauer et al, 2013) and The Positive Emotions Programme for Schizophrenia (Favrod et al., 2019) were developed to emphasise future thinking in terms of goal-setting, and planning, and to anticipate a more positive future. However, they focus more on the practical aspects and applications of utilising EFT, rather than particular training in improving this type of cognitive ability. A series of studies utilising a laboratorybased paradigm of a brief video followed by an interview about details of the video can increase episodic detail for future events, which generalises to improved performance on tasks which follow this induction (Madore, Gaesser, \& Schacter, 2014b, 2016; Madore et al., 2014a, 2015, 2019). These studies demonstrate the important role of episodic detail on a range of tasks; however, the ability to generate higher numbers of specific events is not typically tested, rather, details are assessed within specific events. Further, participants are not consciously aiming to improve episodic thinking skills in this induction, so it is unclear whether any actual learning takes places. Lastly, the longevity of these effects beyond lab testing are unknown, but may be short-lived.

\subsection{The current study}

This study aimed to assess the efficacy of a future training program, Future Specificity Training (FeST), compared to a wait-list control group. The primary outcomes of interest were whether the training intervention would increase (1) the ability to generate specific self-referent future thoughts (spatiotemporally located); (2) the amount of detail in these thoughts; and (3) the use of visual imagery. The secondary outcomes were increases in anticipated and anticipatory pleasure, perceived control, and likelihood of occurrence. Based on past findings, the FeST program might be expected to have effects on these outcomes, which might indicate the utility of such a training program in remediating or enhancing aspects of psychological functioning. Lastly, we were also interested in whether such a training program might have effects on future thinking that is not specific in nature. In particular, we were interested in positive, self-related content that might be of a conceptual or abstracted end state (Barsics, Van der Linden, \& D'Argembeau, 2016), for example, becoming fit or feeling confident. The process of imagining specific future events is guided, constrained, and organized by higher-order autobiographical knowledge (e.g., personal goals and future self-images; D'Argembeau \& Mathy, 2011;
D'Argembeau \& Demblon, 2012). Thus, training to imagine specific future events may also increase the elaboration of higher-order representations of the future, as these are used to imagine specific future events and to organise these in coherent themes and sequences. These representations of a future self are likely to play an important role in helping to organise and guide self-directed behaviour. Therefore, it was also assessed whether the training impacted on such positive conceptual goals in terms of detail, imagery, anticipated and anticipatory pleasure, perceived control, and likelihood of occurrence. It was hypothesised that the future thinking training would lead to significantly higher scores on all the aforementioned outcomes compared to the wait-list group.

\section{Methods}

\subsection{Design}

The study used a between (group: training group; control group) and within-subjects (time-point: baseline, post-training, and follow-up two weeks later) design to test the effects of the training program on the dependent variables. To assess the primary outcomes of EFT specificity, detail, and imagery, and all secondary outcomes, measures were administered at all time-points. Measures of mental health and cognitive functioning were administered at baseline only, to help to describe the sample and assess whether the groups were equivalent on these measures.

\subsection{Participants}

Eligible participants were English-speaking adults ( $\geq 18$ years) who had access to the internet via a laptop or home computer to complete the outcome measures. Participants were recruited via online advertisements distributed through social media (e.g., Facebook, Instagram), university student bodies, and snowballing. A total of 72 participants were recruited and randomized to the two groups. Seven people in the control group only completed the baseline measures, and five people in the future thinking training dropped out after one session citing reasons of being unable to attend a second session in the timeframe of 1-2 weeks $(n=3)$ and finding participation too time-consuming $(n=2)$. This left 60 participants in the final sample, with 32 in the future thinking training and 28 in the waitlist control group. The average age of the sample was 31 years $(S D=13.2$, range 18-67), with $60 \%$ identifying as female, $38.4 \%$ males, and $1.6 \%$ "Other". The majority reported that their highest educational achievement was an undergraduate degree (45\%), then high school completion (30\%), diploma or certificate $(15 \%)$, and postgraduate degree $(10 \%)$. The majority of people (59.3\%) identified as White/Caucasian, 30.5\% Asian, 3.4\% Arab or Middle Eastern, and 6.8\% "Other".

\section{Materials}

Primary Outcomes. The Episodic Future Thinking Test (EFT-T; Hallford, Takano, Raes, \& Austin, 2019) was used to assess the ability to provide specific EFT using cue-word prompts and the subjective detail of EFT and use of mental imagery. The EFT-T has been shown to be a psychometrically robust measure and has a one-factor structure. Participants were presented with eight cue words at each time-point, four positively-valenced and four negatively-valenced, in alternating order. They were instructed to describe an event or activity related to or inspired by the cue word that was either planned to happen, or hypothetically could happen, in their future. They were instructed that this was to be something specific that would occur in the space of one day, and that it was something they would be personally involved in. No time limits were given. Twenty cue words were taken from the original EFT-T word sets (see Hallford et al., 2019), and four cue words were added using the same method of balancing the frequency of word 
use, emotional valence, and intensity (see Appendix A for the cue word sets). Participants were asked to provide as many details that they could and provide a different event/activity for each cue word. Two examples of specific EFTs were provided for the words pleasure and table on a separate webpage to the instructions. Two authors coded a subset of 100 cue word responses (?? ??) as either 1 (specific) or 0 (non-specific) while blinded to condition and inter-rater reliability was found to be acceptable (Cohen's kappa $=.84$ ). The remaining responses were coded by author (??) while blinded to condition. Inspection of the written responses indicated that they were sensical in nature, and there were no repeated responses. Omissions or expressed failures to generate future events to a cue word were marked as non-specific (e.g., "I couldn't think of anything"). Scores were summed for each participant to generate an overall EFT specificity score. The internal reliability was acceptable (Cronbach's $\alpha=0.78$ ). Although participants were not given a timeframe for their future thoughts, they were asked when they imagined this event happening and were given six response options $(1=$ next $24 \mathrm{~h}, 2=$ next week, $3=$ next month, $4=$ next year, $5=$ next 5 years, $6=$ next 10 years or more). The responses to these items were averaged to create an index of temporal distance, or time perspective, when thinking of the future events (Cronbach's $\alpha=0.72$ ).

Once participants had provided each future event, they were then asked to rate them on 1 (Not at All) to 9 (Very Much So) response scales on a series of dimensions. The items were averaged across the cue words to create indices of detail ("How vivid and detailed is your thought of this event/activity?", $\alpha=0.70$ ), and mental imagery ("How much did you find yourself thinking in pictures/mental images about this activity?", $\alpha=0.75$ ).

Secondary Outcomes. Using the same scales as detail and mental imagery in relation to the future events, participants also reported their anticipated pleasure ("How pleasurable/enjoyable do you think it will be to do this event/activity?", $\alpha=0.54$ ), anticipatory pleasure ("How pleasurable/enjoyable is it to just think about doing this event/activity?", $\alpha=0.61$ ), perceived control ("How easy would it be to do this event/activity?", $\alpha=0.57$ ), and perceived likelihood of occurrence ("How likely is it that this event/activity will happen?", $\alpha=0.70$ ). With respect to anticipated and anticipatory pleasure, these were both assessed as they are conceptually distinct constructs (Baumgartner, Pieters, \& Bagozzi, 2008), the former relating to the expectation of pleasure and the latter to an affective response when thinking about the future. Both were assessed given evidence of divergent associations with behavioural intentions and phenomenological characteristics of EFT (e.g., Barsics et al., 2016; Baumgartner et al., 2008).

Items from the Prospective Imagery Test (Stöber, 2000) were used to prompt participants to briefly form a mental image of the following scenarios happening to them: "Things will work out as you hoped", "You will be able to cope easily with pressure", "You will achieve things you set out to do", "You will be very fit and healthy", and "You'll make good and lasting relationships". There were no instructions given about providing specific responses. Participants could think about each scenario for as long or as little as they liked, and they were then asked to complete the same questions posed after the EFT-T cue words, which were also averaged together: detail $(\alpha=0.86)$, mental imagery $(\alpha=0.82)$, anticipated pleasure $(\alpha=0.85)$, anticipatory pleasure ( $\alpha=0.82$ ), perceived control ( $\alpha=0.67$ ), likelihood of occurrence $(\alpha=0.83)$.

Depressive and anxiety symptoms. The self-report depression and anxiety subscales from the 21 -item version Depression, Anxiety, and Stress Scale (DASS-21; Lovibond \& Lovibond, 1995) were used to assess aspects of baseline mental health. Each subscale has seven items referring to core symptoms of the respective negative affective states which are answered on a scale from 0 (Did not apply to me at all) to 3 (Applied to me very much, or most of the time). The internal consistency was acceptable for the depression subscale $(\alpha=0.92)$ and anxiety subscale $(\alpha=0.78)$.

Executive functioning. The Controlled Word Association Test
(COWAT) was used to assess executive functions of selective attention, inhibition, and self-monitoring while retrieving information from memory. The COWAT involves participants providing as many words as they can that start with a particular letter, in this case, "e", while excluding pronouns. A time limit of $60 \mathrm{~s}$ was given, with a timer displayed on the screen before the survey moved to the next page. The number of correct words beginning with the letter e were tallied, excluding any repetitions, minor variations (e.g., egg, eggs), or pronouns (e.g., Emily).

FeST Program. The training program was group-based and comprised two sessions which ran for 90 min each. The training was delivered in a standardised manner, using the Future Specificity Thinking Training Manual developed for this study, with some content adapted from the Memory Specificity Training manual (Dalgleish et al., 2014; Raes, Williams, \& Hermans, 2009). The FeST Manual is available on request from the corresponding author.

The first session involved psychoeducation about EFT and its functions, and distinguishing between general and specific EFT. The facilitators provided examples of generating specific episodic future thoughts in response to cue words. Participants then practised this exercise, using the positive and neutral cue words: bicycle, car, happy, knowledge, with a focus on generating details relevant to the future event (e.g., sensorial and scene details, actions, people, thoughts, feelings etc.), using mental imagery, and imagining events from a firstperson perspective, which is known to be associated with pre-experiencing (D'Argembeau et al., 2012). The responses were then discussed as a group, with facilitators providing individual feedback. The participants were then given more time to provide specific answers to those future thoughts which were not specific, and to add episodic detail to those that were specific. Participants were encouraged to complete a homework task over the next week consisting of practice cue words and providing a daily future thought of something that would or could happen the following day.

The second session began with a brief review of session one and the homework. Further psycho-education about EFT was provided with a focus on anticipating positive emotions. Facilitators provided examples of positive episodic future thoughts in response to cue words, which were contrasted with neutral future thoughts. Participants practised generating two distinct (i.e., one neutral and one positive) episodic future thoughts per word using the cue words: success, book, justice, money, respect, hand, brave, and house. When generating positive future thoughts, participants were encouraged to focus on the emotions they expected to experience. Participants shared their mental simulations with the group, were asked to describe the difference between the two future thoughts, and received feedback from the facilitators. Finally, participants were encouraged to complete another homework task that was comparable to worksheet one, but required participants to generate two specific episodic future thoughts per word.

Participant perceptions and attitudes towards the training. A series of response scales rated from 1 to 7 were used to assess participants' perception and attitudes towards the future thinking training in terms of how easy they found it to understand, how helpful they thought it was, whether it was long enough, and whether they would recommend it to others. Responses indicating disagreement ( 1 = Strongly Disagree, 2 = Disagree, 3 = Somewhat Disagree $)$ were combined to form a disagreement index, and responses indicating agreement (5 = Somewhat Agree, $6=$ Agree, and $7=$ Strongly Agree $)$ were combined to indicate an agreement index. A response of 4 indicated neither agreement nor disagreement.

\subsection{Procedure}

The study was approved by the University Human Research Ethics Committee. Potential participants followed a link to a description of the study, and after reading and providing informed consent they were randomly allocated to either the training or wait-list control group using computerised, simple randomisation and emailed a link to the 
baseline questionnaire. All questionnaires were presented on an online survey platform, and took an estimated $45 \mathrm{~min}$ to complete. Once the baseline questionnaire was completed, participants were contacted to schedule a time for the first session. One or two co-facilitators, contingent on group size, delivered the sessions online through video conferencing with up to six participants. All four facilitators were students in an approved fourth year of study in psychology, and were supervised by the lead author, a registered clinical psychologist. Following the first session, participants were emailed Worksheet 1 and scheduled to attend Session 2 of the training, occurring approximately one week later. After completing Session 2 they were emailed the second worksheet and completed the post-training survey. Participants in the waitlist condition received no intervention and completed the post-training questionnaires approximately one week following baseline measurement, and the follow-up questionnaires two weeks later. All participants received a $\$ 10$ retail voucher for their participation, and those in the waitlist group had the option to partake in training sessions following completion of the study.

\subsection{Data analytic strategy}

An a priori power analysis was conducted using $G *$ Power software. To estimate an effect size we drew from research on outcomes of Memory Specificity Training, which generally show a large effect on specificity when compared to a wait-list (Barry et al., 2019). It was estimated that at least 21 participants would be needed in each arm to power the study to detect a large (Cohen's $d=0.80$ ) between-group effect at post-training and follow-up using an independent samples $t$ test with alpha $=.05$ and .80 power. Given the experimental nature of this training program, and in order to mitigate against the risk of a type II error, some oversampling was done.

All analyses were conducted using IBM SPSS 25.0. Means and standard deviations were generated for variables at each time point. Baseline comparisons were made using independent samples $t$-tests, and the correlations between variables were examined using Spearman rank correlations. To test the main hypotheses, two-way (group by time of assessment) mixed ANOVAs were used with follow-up independent samples $t$-tests to compare for group differences at post-training and follow-up time points. To correct for multiple tests, the false discovery rate procedure was applied to all tests of interaction effects and followup $t$-tests (Benjamini \& Hochberg, 1995). This procedure aims to control the proportion of significant results that are type I errors. A corrected significance level is provided after the procedure $(q)$, with all $p$ values less than 0.027 considered to be statistically significant at the $\alpha=0.05$ level.

\section{Results}

\subsection{Preliminary analyses}

There was no significant differences between the groups on age, $t$ $(57)=1.7, p=.084$, self-identified gender, $\chi 2(2)=2.9, p=.233$, educational achievement, $\chi 2(3)=0.1, p=.991$, or ethnicity, $\chi^{2}$ (3) $=1.1, p=.777$. Table 1 shows the means and standard deviations for all study variables. No significant differences were found between the groups at baseline on any of the outcome measures ( $t$ 's all $<1.97$, all $p$ 's $>0.054)$. There was no difference between the groups on DASS21 depressive $(M=4.2, S D=3.7)$ and DASS-21 anxiety symptoms $(M=4.2, S D=3.4), t^{\prime} s<1.1, p$ 's $\left.>0.299\right)$, with the mean scores indicating low levels of symptoms on average in this sample, and a narrow distribution of scores. The mean score on the COWAT was 10 $(S D=2.5)$, with no difference between the groups, $t(58)=0.12$, $p=.903$.

At baseline, future events on the EFT-T items were, on average, imagined as happening between the next month and the next year $(M=3.4, S D=0.82)$. A mixed ANOVA showed an interaction effect, $F$
$(2,116)=5.5, p=.005, \eta_{p}^{2}=0.08$. Although the groups did not significantly differ at post-training and follow-up (both $t<1.3$, $p>.230$ ), within-group paired samples $t$-tests showed the training group reported imagining future events as significantly closer in time at follow-up compared to baseline, $t(32)=4.1, p<.001, d=0.82$, whereas the control group did not change, $t(28)=0.08, p=.936$, $d=0.01$.

Table 2 shows a correlation table of the EFT-T measures at baseline. EFT-T specificity was found to correlate with detail, imagery, and perceived control. The DASS-21 depression scores correlated negatively with anticipatory pleasure and perceived likelihood. Executive functioning assessed by the COWAT correlated significantly with EFT-T detail, imagery, and perceived control. It is noted that the sample size was too small to detect associations of a small magnitude. The remaining EFT-T variables correlated moderately, whilst anticipated and anticipatory pleasure correlated strongly.

Participant perceptions and attitudes towards the training. Twenty-two of the participants provided feedback on the training $(68.8 \%)$. Of them, $95.5 \%$ agreed the training was easy to understand (4.5\% disagreed), $86.4 \%$ agreed it was helpful (13.6\% neither agreed nor disagreed), $68.2 \%$ thought it was long enough $(18.2 \%$ neither agreed nor disagreed, and $13.6 \%$ disagreed) and $72.7 \%$ would recommend it to others (18.2\% neither agreed nor disagreed, and $9.1 \%$ ).

\subsection{Primary outcomes on specificity, detail, and imagery}

We focus here on the ANOVA interaction effects (group $\mathrm{x}$ timepoints) which are relevant to the study hypotheses. A mixed ANOVA for specificity on the EFT-T showed a significant interaction effect, $F(2$, $116)=10.9, p<.001, \eta_{p}^{2}=0.15$. Fig. 1 shows the scores over timepoints. Follow-up $t$-tests showed a small, but non-significant mean difference in favour of the training group at post-training, $t(58)=0.9$, $p=.371, d=0.23$, with this difference becoming large and significant at the follow-up, $t(58)=3.2, p=.002, d=0.82$. An interaction effect was found for self-reported detail on the EFT-T, $F(2,116)=11.9$, $p<.001, \eta_{p}^{2}=0.17$, with a small, non-significant mean difference in favour of the training group at post-training, $t(58)=1.0, p=.309$, $d=0.26$, becoming large and significant at the follow-up, $t(58)=5.1$, $p<.001, d=1.32$ (see Fig. 2). An interaction effect was also found for self-reported visual imagery on the EFT-T, $F(2,116)=6.2$, $p=.003, \eta_{p}^{2}=0.09$, and follow-up $t$-tests again showed a small, nonsignificant mean difference in favour of the training group at posttraining, $t(58)=0.8, p=.426, d=0.20$, with this difference becoming large and significant at follow-up, $t(58)=4.4, p<.001$, $d=1.33$ (see Fig. 3).

\subsection{Secondary outcomes on anticipated pleasure, anticipatory pleasure, perceived control, and likelihood of occurrence}

Interaction effects were found for anticipated pleasure, $F(2$, 116) $=7.2, p<.001, \eta_{p}^{2}=0.15$, and anticipatory pleasure, $F(2$, 116) $=7.0, p<.001, \eta_{p}^{2}=0.14$, on the EFT-T. Follow-up tests showed that the groups did not significantly differ at post-training (both $t<1.49, p>.143, d<0.38$ ), but scores were significantly higher in the training group at follow-up on anticipated pleasure, $t(58)=7.0$, $p<.001, d=1.78$, and anticipatory pleasure, $t(58)=4.2, p<.001$, $d=1.07$. An interaction effect was found for perceived control on the EFT-T, $F(2,116)=4.9, p=.009, \eta_{p}^{2}=0.07$. Follow-up $t$-tests showed a small, non-significant difference in mean scores in favour of the training group at post-training, $t(58)=1.2, p=.224, d=0.32$, which was large and significant at the follow-up, $t(58)=4.6, p<.001, d=1.20$. For perceived likelihood of the event happening there was significant interaction effect, $F(2,116)=4.9, p=.003, \eta_{p}^{2}=0.09$, with a trivial, non-significant group difference at post-training, $t(58)=0.5, p=.611$, $d=0.13$, but a large, significant difference in favour of the training 
Table 1

Means and standard deviations for the study variables.

\begin{tabular}{|c|c|c|c|c|c|c|}
\hline \multirow[b]{2}{*}{ Variable } & \multicolumn{3}{|c|}{$\begin{array}{l}\text { Intervention }(N=32) \\
\mathrm{M}(S D)\end{array}$} & \multicolumn{3}{|c|}{$\begin{array}{l}\text { Control }(N=28) \\
\mathrm{M}(S D)\end{array}$} \\
\hline & Baseline & Post-Training & Follow-up & Baseline & Post-Training & Follow-up \\
\hline EFT-T Detail & $6.23(1.21)$ & $6.57(1.27)$ & $7.36(0.77)$ & $6.54(1.11)$ & $6.22(1.37)$ & $6.14(1.04)$ \\
\hline EFT-T Imagery & $6.23(1.47)$ & $6.50(1.69)$ & $7.37(0.67)$ & $6.61(1.27)$ & $6.17(1.58)$ & $6.44(0.97)$ \\
\hline EFT-T Anticipated Pleasure & $4.52(5.92)$ & $5.16(1.07)$ & $6.52(0.24)$ & $4.66(0.67)$ & $4.66(1.50)$ & $5.27(0.97)$ \\
\hline EFT-T Anticipatory Pleasure & $4.37(0.81)$ & $4.83(1.12)$ & $6.02(0.83)$ & $4.81(0.94)$ & $4.43(1.50)$ & $5.10(0.85)$ \\
\hline EFT-T Perceived Control & $5.66(0.84)$ & $5.87(1.53)$ & $7.01(0.76)$ & $5.80(1.51)$ & $5.42(1.25)$ & $6.00(0.91)$ \\
\hline EFT-T Likelihood & $6.24(1.35)$ & $6.90(1.02)$ & $7.32(0.60)$ & $6.66(1.07)$ & $6.76(1.05)$ & $6.60(0.72)$ \\
\hline PIT Detail & $6.53(1.57)$ & $6.68(1.14)$ & $7.50(0.98)$ & $6.48(1.70)$ & $6.76(1.59)$ & $6.41(1.52)$ \\
\hline PIT Imagery & $6.34(1.51)$ & $6.65(1.30)$ & $6.83(1.84)$ & $6.39(1.72)$ & $6.44(1.76)$ & $6.23(1.41)$ \\
\hline PIT Anticipated Pleasure & $8.23(0.84)$ & $8.10(1.02)$ & $8.50(0.58)$ & $8.02(0.88)$ & $8.20(0.26)$ & $7.88(0.80)$ \\
\hline PIT Anticipatory Pleasure & $6.98(1.39)$ & $7.65(1.18)$ & $8.28(0.55)$ & $6.87(1.49)$ & $7.81(1.08)$ & $7.71(0.54)$ \\
\hline PIT Perceived Control & $6.47(1.05)$ & $6.90(0.95)$ & $7.08(0.63)$ & $6.84(1.07)$ & $7.01(1.14)$ & $6.71(0.92)$ \\
\hline PIT Likelihood & $6.41(1.45)$ & $6.66(1.05)$ & $6.76(1.12)$ & $6.56(1.31)$ & $6.80(1.21)$ & $6.29(1.41)$ \\
\hline
\end{tabular}

EFT-T $=$ Episodic Future Thinking-Test, PIT $=$ Prospective Imagery Test .

Table 2

Correlations of EFT-T variables at baseline.

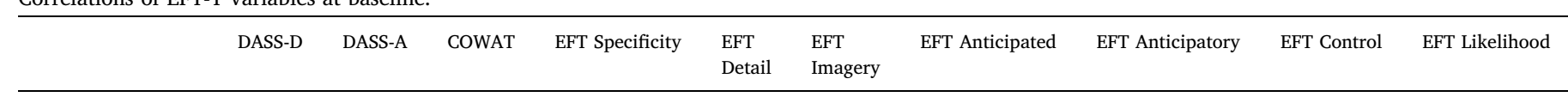

\begin{tabular}{|c|c|c|c|c|c|c|c|c|c|c|}
\hline DASS-D & - & & & & & & & & & \\
\hline DASS-A & $.56^{* * *}$ & - & & & & & & & & \\
\hline COWAT & -.07 & -.06 & - & & & & & & & \\
\hline EFT-T Specificity & -.08 & -.09 & .12 & - & & & & & & \\
\hline EFT-T Detail & -.10 & -.10 & $.26^{*}$ & $.42 * *$ & - & & & & & \\
\hline EFT-T Imagery & -.06 & -.02 & $.37^{* *}$ & $.39 * *$ & $.78^{* * *}$ & - & & & & \\
\hline EFT-T Anticipated & -.19 & -.02 & .08 & .03 & $.36^{* *}$ & $.34 * *$ & - & & & \\
\hline EFT-T Anticipatory & $-.25^{*}$ & -.04 & .10 & -.13 & $.35^{* *}$ & $.30 *$ & $.76^{* *}$ & - & & \\
\hline EFT-T Control & -.07 & .00 & $.25^{*}$ & $.41^{* *}$ & $.36 * *$ & $.44^{* *}$ & .26 & .12 & - & \\
\hline EFT-T Likelihood & $-.26^{*}$ & -.13 & -.03 & .13 & $.36^{* *}$ & $.32 *$ & $.34^{* *}$ & $.31 *$ & .27 & - \\
\hline
\end{tabular}

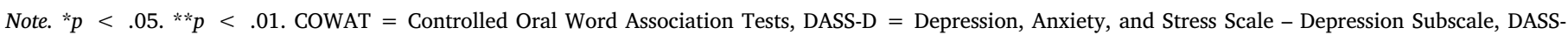
$\mathrm{A}=$ Depression, Anxiety, and Stress Scale - Anxiety Subscale, EFT-T = Episodic Future Thinking-Test.

group at the follow-up, $t(58)=4.2, p<.001, d=1.09$.

\subsection{Outcomes on positive, generalised future self-states}

The results for the PIT items showed significant interaction effects for detail, anticipated pleasure, and perceived control (all $F^{\prime} s>3.3$, all $p$ 's $<0.012)$, a trend for anticipatory pleasure $(F=3.3, p=.045)$ and likelihood $(F=2.4, p=.088)$, but a clearly non-significant result for imagery $(F=1.2, p=.284)$. Follow-up $t$-tests for detail, anticipated pleasure, anticipatory pleasure, and perceived control showed no group differences at post-training (all $t^{\prime}$ s $<.06$, all $p$ 's $>0.586$ ), but there were significant differences at follow-up, with higher scores in the training group on detail, anticipatory pleasure, and anticipated pleasure (all $t$ 's $>3.3, p$ 's $<0.003, d$ 's $=0.84-1.06$ ), and a trend towards higher scores for perceived control, $t(58)=1.8, p=.075, d=0.47$.

\section{Discussion}

This study was the first to examine a training program aimed to improve specific EFT in a community sample. The results show that a two-session training program can have effects on the specificity, level of detail, and imagery of EFT. Significant effects were observed at a twoweek follow-up, but not at the post-training measures administered immediately following the second session. This may be because the two training sessions were spaced only one week apart, leaving little time for skills acquisition, especially that occurring in the second session, to take effect. Further, participants were provided with homework to complete after the second session and were encouraged to practice simulating specific EFT in daily life. Both factors might have caused further improvements. The effects at follow-up were generally large and provide evidence that people can be trained to improve their ability to provide spatiotemporally located simulations of future events, and subjective experience of detail and use of visual imagery in future thinking. Of the participants that provided feedback on the training, the majority found it easy to understand, helpful, and would recommend it to others. Interestingly, some participants did not agree that it was long enough, suggesting interest from a subsample of participants in a longer series of training sessions.

At follow-up, participants in the training group reported having more perceived control over the EFT-T future events that they described and perceived them as more likely to happen. This supports previous research (Boland et al., 2018; Taylor et al., 1998; Brownet al., 2002) indicating that enhancing EFT might be one method to making future events feel more under one's control, and as being more likely to occur. Anticipated and anticipatory pleasure were also significantly increased in the training group compared to the control group at follow-up, indicating that participants felt significantly more positive emotion in regards to what the events would be like to experience and what they were like to think about when completing the EFT-T.

On the PIT items that represented positive future states, increases in detail, anticipated pleasure, and anticipatory pleasure were found. These results are roughly consistent with those from the EFT-T. The lack of change in mental imagery might be due to it being harder to use visual imagery for conceptual thoughts such as these, which might involve verbal or symbolic mental representations rather than contextual, concretely defined content. Relatedly, while perceived control and likelihood trended in the expected direction, the current study was not powered to detect group differences that appeared to be only moderate in magnitude. These findings indicate that it might be more difficult to shift expectations in relation to abstracted future circumstances that are 


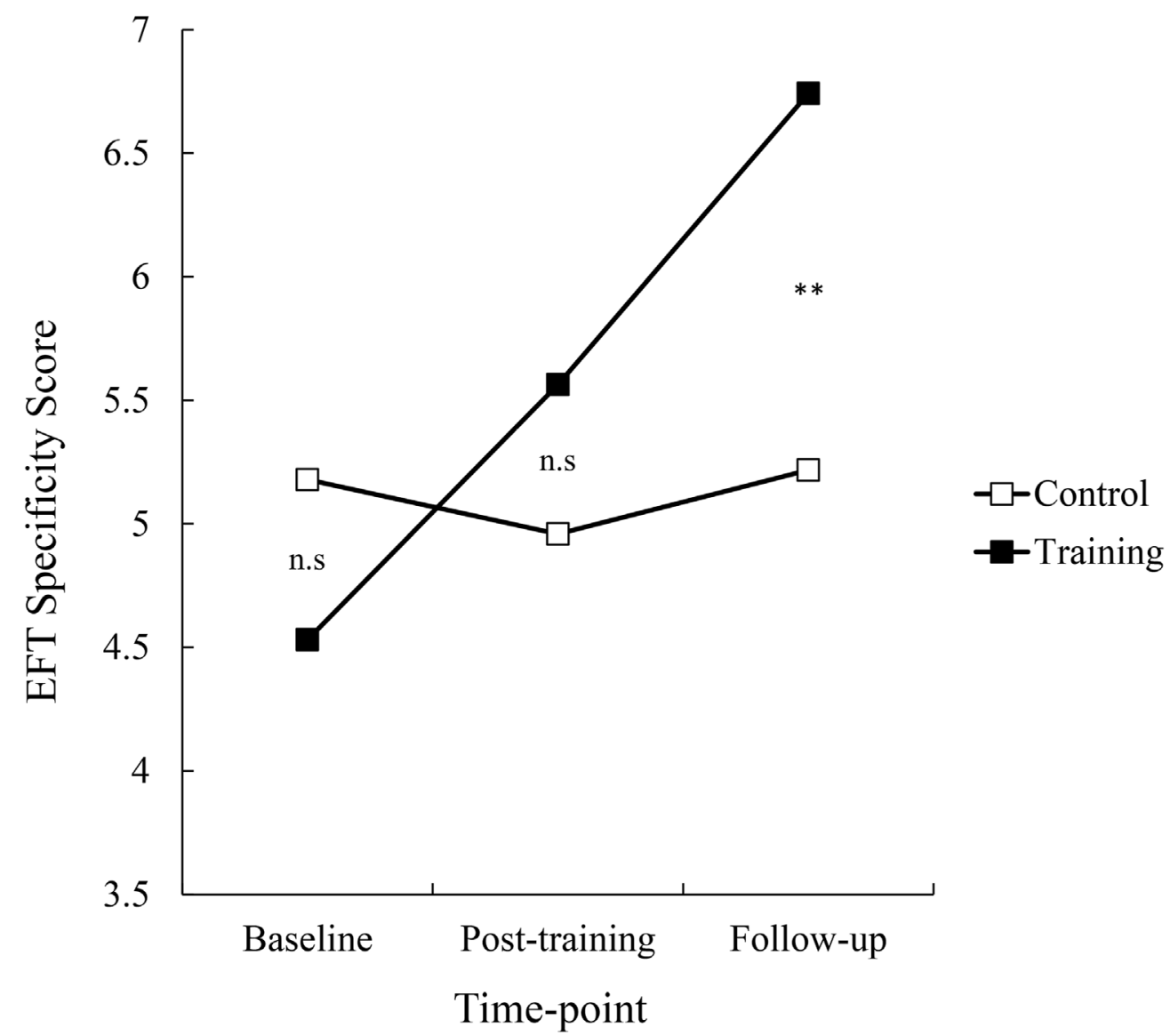

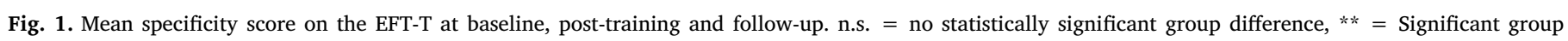
difference at the $p<.01$ level.

not necessarily tied to specific events. Explicit instructions to imagine specific examples of positive future states may be needed to increase perceived control and likelihood of occurrence, such as in Boland et al. (2018) study where similar prompt items were used, but participants were asked to imagine spatiotemporally located examples (e.g., a specific example of being successful). These results provide evidence that training in specific EFT can have generalised, large effects on thinking of abstracted positive future states in terms of detail and positive emotion, but if there are effects on perceived control and likelihood of occurrence, they may only be moderately-sized in magnitude.

The FeST program could be extended to incorporate content on the application of future thinking. For example, applying these skills to planning behaviours, problem-solving, goal setting, or decision-making tasks within sessions might help individuals to consciously utilise future thinking in particular ways for practical outcomes. Laboratory studies have indicated such effects on tasks following inductions to increase episodic detail (Madore et al., 2014a; Madore and Schacter, 2014b etc), although this was not posited as an explicit goal for participants. It may be interesting to see how effects on EFT generalise to behaviours in daily life when improving episodic thinking is a conscious and effortful task. The program could also be extended to integrate the distinction between imagining future outcomes and simulating the process needed to attain these outcomes (Taylor et al., 1998). Indeed, there is evidence that indulging in positive fantasies about desired futures can have negative consequences, reducing the effort invested in actually realizing these futures (Oettingen, Mayer, \& Portnow, 2016). Techniques that help people to place desired future states in perspective with a clear sense of reality, such as mental contrasting (Oettingen et. al, 2016), could thus fruitfully complement our specificity training.

This training program could be used to remediate impairments in future thinking in psychiatric disorders and help relieve symptoms through disrupting psychopathological processes. For example, improving EFT in posttraumatic stress disorder may lead to improvements in self-efficacy, which is impoverished in PTSD, but amenable to change (Brown et al., 2016). One caveat here is that increased perceived control and likelihood of occurrence of events may not always be advantageous, given that some events (both positive and negative) might be beyond one's control or highly unlikely to occur. In this context, it would be useful to consider how future thinking could facilitate more realistic appraisals of future events and their consequences. In particular, this may be of use in conventional cognitive-behavioural techniques such as cognitive restructuring or imaginal exposure. Another example of use might be in generalised anxiety disorder (GAD). The cognitive avoidance theory suggests that the excessive worrying that characterises GAD is maintained by avoidance of unpleasant emotional and physical responses through using less detail and mental imagery for possible negative events (Borkovec, Alcaine, \& Behar, 2004). This may then lead to a failure in adaptive emotional and informational processing, such as identifying how a potential outcome may be emotionally experienced, or practically influenced or overcome. Some recent research has indicated that increasing the use of mental imagery might disrupt the occurrence of worry (Skodzik, Leopold, \& Ehring, 2017, 2018), suggesting that specificity and detail in thinking may be key targets to focus on. FeST may be a suitable program to test for this purpose. Alternatively, the contrast avoidance mode of worry (Newman \& Llera, 2011) suggests that worry is used to maintain a negative emotional state in order to avoid unexpected emotions shifts or contrasts in emotional experience. FeST may also be useful in terms of helping people to learn to shift from negative to positive future experiences and develop flexibility in emotional experience. Future 


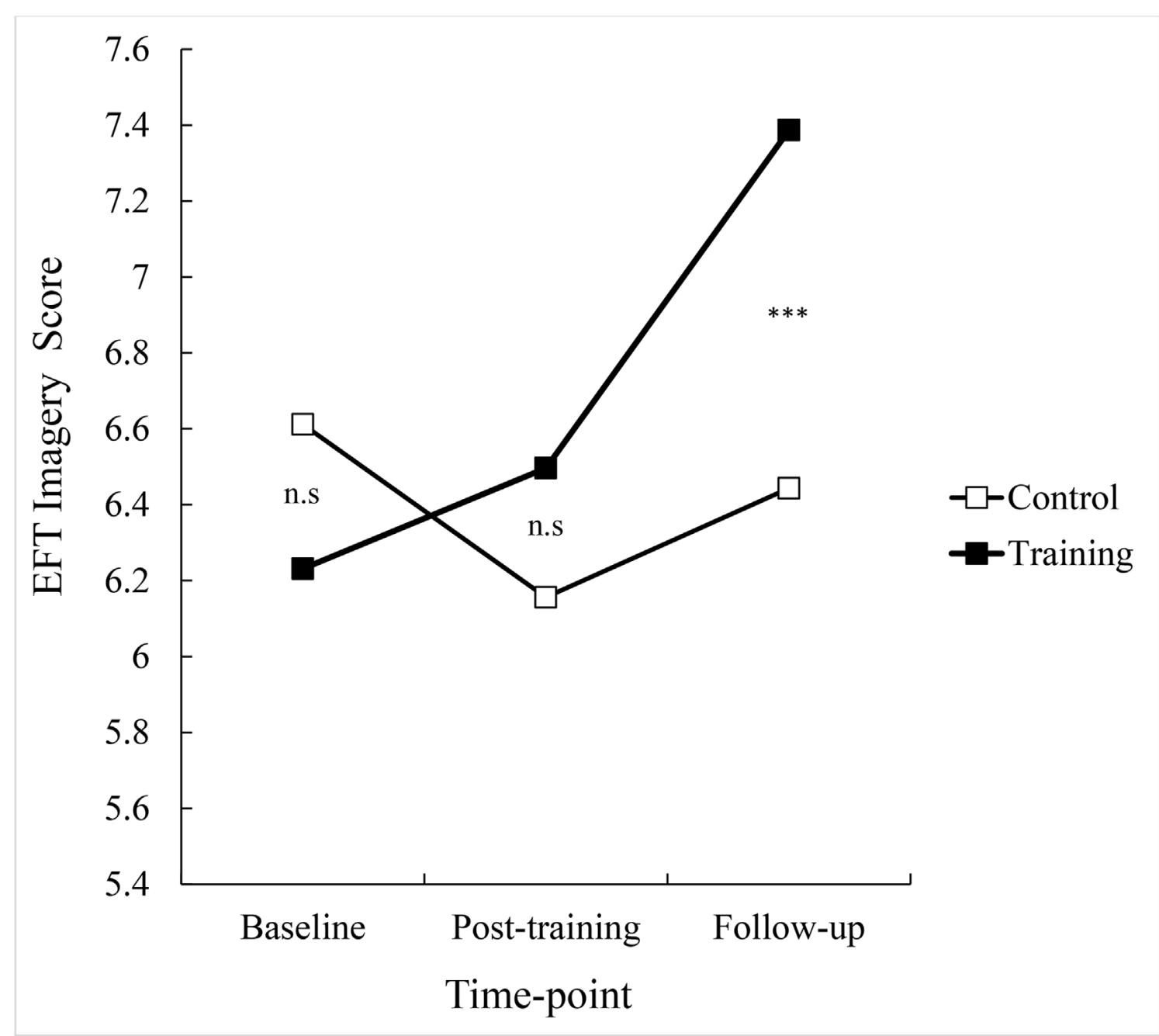

Fig. 2. Mean detail score on the EFT-T at baseline, post-training and follow-up. n.s. $=$ no statistically significant group difference, $* * *=$ Significant group difference at the $p<.001$ level.

studies may test whether it helps reduce worry and through which pathways. The effects on anticipated and anticipatory pleasures are noteworthy as these are diagnostic and phenomenological hallmarks of depression and schizophrenia-spectrum disorders (Hallford \& Sharma, 2019). In particular, this program could be tested in a depressed sample, where therapeutic options for lack of pleasure/anhedonia are strongly needed (Calabrese et al., 2014), as it predicts a less favourable course of depression and poorer response to treatment (Dunn \& Roberts, 2016). The findings on anticipated and anticipatory pleasure complement previous findings of the associations between EFT characteristics and prospective emotions (Hallford, 2019; Hallford, Takano, Raes, \& Austin, 2019, 2020), and indicate a standardised method that might be used to target anhedonia. Incorporating this training into validated interventions that focus on future events, such as behavioural activation (Ekers et al., 2014) or problem-solving therapy (Cuijpers, van Straten, \& Warmerdam, 2007), might incrementally improve outcomes by helping increase anticipation of reward, motivation (see Renner, Ji, Pictet, Holmes, \& Blackwell, 2017 , 2017 for an example of future thinking to 'amplify' motivation), and engagement in adaptive behaviour.

The sample was recruited from the community and was, on average, in reasonable mental health on our indicators. Therefore, how the training program and its outcomes would generalise to other samples with impairments in episodic thinking is unknown. While this study indicates that individuals without formal training in professional psychology can deliver FeST in community samples, clinical skills may be needed depending on the characteristics of the group. The follow-up period was two weeks, and it is not known whether any effects would be maintained for longer periods. It is not known what caused the dropout of participants. Although small in number, they may represent participants that did not find the training useful or easy to understand, or received no benefit, therefore positively biasing the evaluation questions/feedback. The EFT-T cue word sets were not counterbalanced across conditions. Although this introduces the possibility that differences across time-points were affected by qualities of the word sets, these word sets were matched on several dimensions, and our research has shown that this process leads to negligible differences in mean scores (Hallford et al., 2019). It is possible that repeated simulations on the same PIT items that were used at each time-point could have caused effects on detail and other measures, as shown in previous research (Szpunar \& Schacter, 2013 , May). However, given that both groups simulated the same future events at each time-point, but only the training group reported significantly higher scores, we can rule this out as a causal factor. An important limitation, generally applicable to studies employing subjective self-report measures, is the possibility of demand characteristics. Knowledge of the general aims of the study might have contributed to higher ratings on some self-report measures relating to imagined events. Efforts were made to reduce this by asking participants to answer as honestly as possible at the start of each survey, and they were not made aware of our specific hypotheses. In relation to objectively rated specificity, research has indicated that there are also 


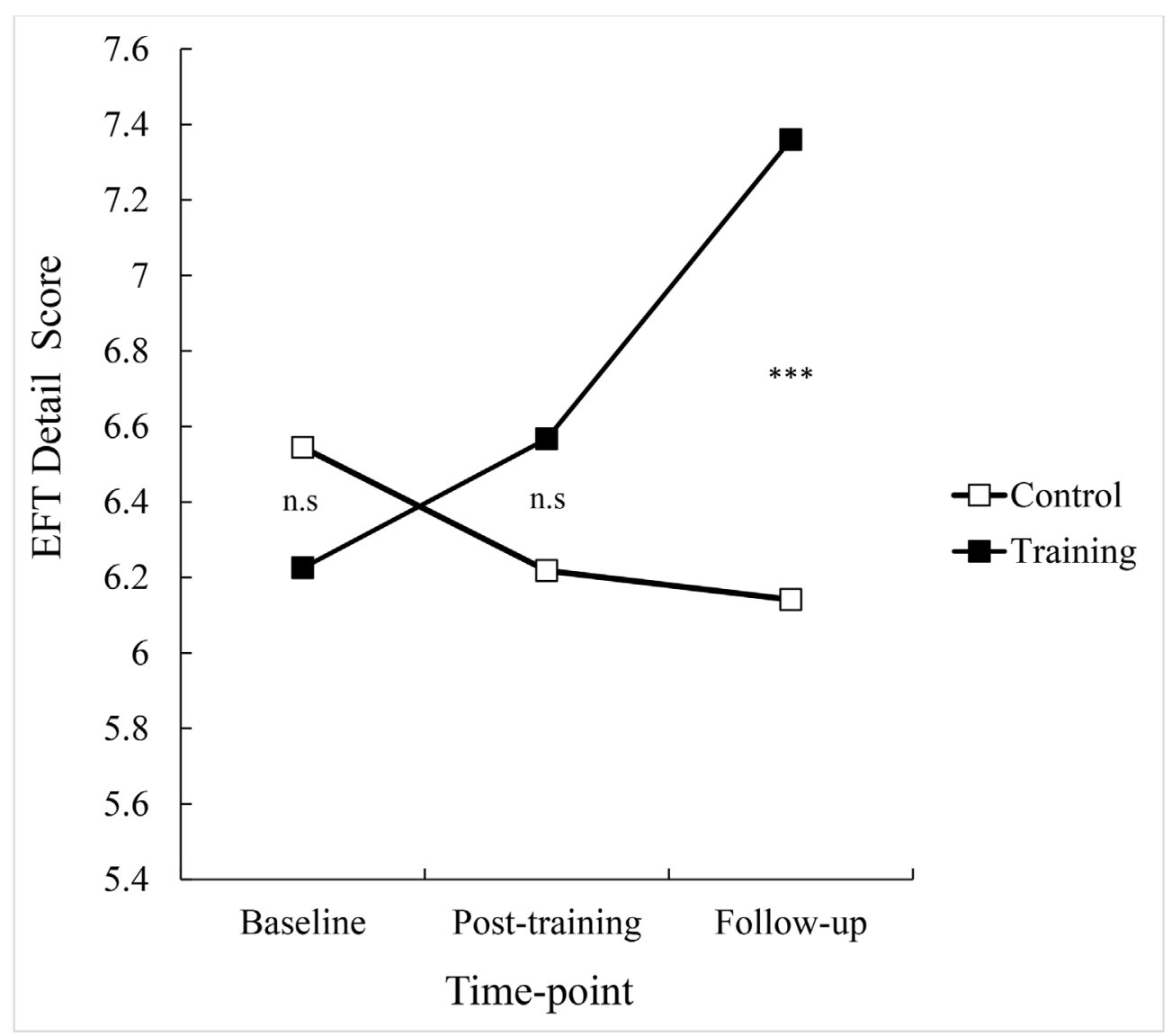

Fig. 3. Mean imagery score on the EFT-T at baseline, post-training and follow-up. n.s. $=$ no statistically significant group difference, $* * *=$ Significant group difference at the $p<.001$ level.

changes in implicit measures of memory specificity following specificity training (Raes et al., 2009), suggesting that changes may not be just related to increased motivation.

FeST is designed to improve dimensions of EFT; however, given the overlap with other forms of episodic thinking, such as autobiographical memory and counterfactual thinking, it may also affect these. Future studies might assess the effects of FeST using measures of specificity, detail and imagery in other forms of episodic thinking. A head-to-head trial of MeST and FeST, which both target episodic thinking, might be interesting to assess whether or not they have unique effects on past and future thinking. This might be especially interesting in the context of disorders such as schizophrenia in which past and future thinking specificity appear to be somewhat dissociated (Barry, Hallford, Del Ray, \& Ricarte, 2019).

In summary, these findings indicate that FeST, a manualised training program in EFT, can cause increases in the ability to mentally simulate thoughts of specific, spatiotemporally-located future events, along with more detail and use of mental imagery. These effects are accompanied by increases in anticipated and anticipatory pleasure, perceived control, and likelihood of occurrence, and some generalised effects on imagining abstracted positive future self-states.

\section{Declarations of interest}

None.

\section{Funding}

This research did not receive any specific grant from funding agencies in the public, commercial, or not-for-profit sectors.

\section{CRediT authorship contribution statement}

D.J. Hallford: Conceptualization, Methodology, Formal analysis, Investigation, Writing - original draft. J.J.E. Yeow: Investigation, Resources, Writing - review \& editing. G. Fountas: Investigation, Resources, Writing - review \& editing. C.A. Herrick: Investigation, Resources, Writing - review \& editing. F. Raes: Investigation, Resources, Writing - review \& editing. A. D'Argembeau: Methodology, Writing - review \& editing.

\section{Appendix A}

Cue Word Sets

1) happy, lost, strong, trouble, beautiful, failure, easy, fear

2) hope, stress, truth, pressure, agreement, pain, respect, danger

3) freedom, cold, success, evil, friend, lie, bright, alone

\section{References}

Atance, C. M., \& O'Neill, D. K. (2001, Dec). Episodic future thinking. Trends in Cognitive Sciences, 5(12), 533-539. https://doi.org/10.1016/s1364-6613(00)01804-0.

Barry, T. J., Hallford, D. J., Del Rey, F., \& Ricarte, J. J. (2019, Oct). Differential associations between impaired autobiographical memory recall and future thinking in people with and without schizophrenia. British Journal of Clinical Psychology. https:// doi.org/10.1111/bjc.12236.

Barry, T. J., Sze, W. Y., \& Raes, F. (2019, 05). A meta-analysis and systematic review of Memory Specificity Training (MeST) in the treatment of emotional disorders. Behaviour Research and Therapy, 116, 36-51. https://doi.org/10.1016/j.brat.2019.02. 001.

Barsics, C., Van der Linden, M., \& D'Argembeau, A. (2016). Frequency, characteristics, and perceived functions of emotional future thinking in daily life. Quarterly Journal of Experimental Psychology, 69(2), 217-233. https://doi.org/10.1080/17470218.2015. 1051560. 
Baumgartner, H., Pieters, R., \& Bagozzi, R. P. (2008). Future-oriented emotions: Conceptualization and behavioral effects. European Journal of Social Psychology, 38(4), 685-696. https://doi.org/10.1002/ejsp.467.

Benjamini, Y., \& Hochberg, Y. (1995). Controlling the false discovery rate: A practical and powerful approach to multiple testing. Journal of the Royal Statistical Society: Series B (Methodological), 57(1), 289-300. https://doi.org/10.1111/j.2517-6161.1995. tb02031.x.

Boland, J., Riggs, K. J., \& Anderson, R. J. (2018, 01). A brighter future: The effect of positive episodic simulation on future predictions in non-depressed, moderately dysphoric \& highly dysphoric individuals. Behaviour Research and Therapy, 100, 7-16. https://doi.org/10.1016/j.brat.2017.10.010.

Borkovec, T. D., Alcaine, O. M., \& Behar, E. (2004). Avoidance theory of worry and generalized anxiety disorder. In R. G. Heimberg, C. L. Turk, \& D. S. Mennin (Eds.). Generalized anxiety disorder: Advances in research and practice (pp. 77-108). Guilford Press.

Brown, A. D., Kouri, N. A., Rahman, N., Joscelyne, A., Bryant, R. A., \& Marmar, C. R. (2016). Enhancing self-efficacy improves episodic future thinking and social-decision making in combat veterans with posttraumatic stress disorder. Psychiatry Research, 242, 19-25. https://doi.org/10.1016/j.psychres.2016.05.026.

Brown, G. P., Macleod, A. K., Tata, P., \& Goddard, L. (2002). Worry and the simulation of future outcomes. Anxiety, Stress \& Coping, 15(1), 1-17. https://doi.org/10.1080/ 10615800290007254.

Calabrese, J. R., Fava, M., Garibaldi, G., Grunze, H., Krystal, A. D., Laughren, T., et al. (2014). Oct). Methodological approaches and magnitude of the clinical unmet need associated with amotivation in mood disorders. Journal of Affective Disorders, 168, 439-451. https://doi.org/10.1016/j.jad.2014.06.056.

Cuijpers, P., van Straten, A., \& Warmerdam, L. (2007, Jan). Problem solving therapies for depression: A meta-analysis. European Psychiatry, 22(1), 9-15. https://doi.org/10. 1016/j.eurpsy.2006.11.001.

D'Argembeau, A., \& Demblon, J. (2012, Nov). On the representational systems underlying prospection: Evidence from the event-cueing paradigm. Cognition, 125(2), 160-167. https://doi.org/10.1016/j.cognition.2012.07.008.

D'Argembeau, A., Lardi, C., \& Van der Linden, M. (2012). Self-defining future projections: Exploring the identity function of thinking about the future. Memory, 20(2), 110-120. https://doi.org/10.1080/09658211.2011.647697.

D'Argembeau, A., \& Mathy, A. (2011, May). Tracking the construction of episodic future thoughts. Journal of Experimental Psychology: General, 140(2), 258-271. https://doi. org/10.1037/a0022581.

Dalgleish, T., Bevan, A., McKinnon, A., Breakwell, L., Mueller, V., Chadwick, I., et al. (2014, Jul). A comparison of MEmory specificity training (MEST) to education and support (ES) in the treatment of recurrent depression: Study protocol for a cluster randomised controlled trial. Trials, 15, 293. https://doi.org/10.1186/1745-6215-15293.

Daniel, T. O., Stanton, C. M., \& Epstein, L. H. (2013a). The future is now: Comparing the effect of episodic future thinking on impulsivity in lean and obese individuals. Appetite, 71, 120-125. https://doi.org/10.1016/j.appet.2013.07.010.

Daniel, T. O., Stanton, C. M., \& Epstein, L. H. (2013b). The future is now: Reducing impulsivity and energy intake using episodic future thinking. Psychological Science, 24(11), 2339-2342. https://doi.org/10.1177/0956797613488780.

Dunn, B. D., \& Roberts, H. (2016). Improving the capacity to treat depression using talking therapies. The Wiley Handbook of Positive Clinical Psychology183-204.

Ekers, D., Webster, L., Van Straten, A., Cuijpers, P., Richards, D., \& Gilbody, S. (2014). Behavioural activation for depression; an update of meta-analysis of effectiveness and sub group analysis. PloS One, 9(6), e100100. https://doi.org/10.1371/journal.pone. 0100100.

Engel, M., Fritzsche, A., \& Lincoln, T. M. (2013, Dec). Anticipatory pleasure and approach motivation in schizophrenia-like negative symptoms. Psychiatry Research, 210(2), 422-426. https://doi.org/10.1016/j.psychres.2013.07.025.

Favrod, J., Nguyen, A., Chaix, J., Pellet, J., Frobert, L., Fankhauser, C., et al. (2019). Improving pleasure and motivation in schizophrenia: A randomized controlled clinical trial. Psychotherapy and Psychosomatics, 88(2), 84-95. https://doi.org/10.1159/ 000496479.

Foussias, G., Mann, S., Zakzanis, K. K., van Reekum, R., Agid, O., \& Remington, G. (2011, Oct). Prediction of longitudinal functional outcomes in schizophrenia: The impact of baseline motivational deficits. Schizophrenia Research, 132(1), 24-27. https://doi. org/10.1016/j.schres.2011.06.026.

Hallford, D. J. (2019, 03). The phenomenological characteristics of autobiographical future thinking in dysphoric and non-dysphoric individuals. Psychiatry Research, 273, 481-486. https://doi.org/10.1016/j.psychres.2018.12.100.

Hallford, D. J., Austin, D. W., Takano, K., \& Raes, F. (2018, 03). Psychopathology and episodic future thinking: A systematic review and meta-analysis of specificity and episodic detail. Behaviour Research and Therapy, 102, 42-51. https://doi.org/10. 1016/j.brat.2018.01.003.

Hallford, D. J., Barry, T. J., Austin, D. W., Raes, F., Takano, K., \& Klein, B. (2020, Jan). Impairments in episodic future thinking for positive events and anticipatory pleasure in major depression. Journal of Affective Disorders, 260, 536-543. https://doi.org/10. 1016/j.jad.2019.09.039.

Hallford, D. J., Farrell, H., \& Lynch, E. (2020). Increasing anticipated and anticipatory pleasure through episodic thinking. Emotion (in press).

Hallford, D. J., \& Sharma, M. K. (2019, Nov). Anticipatory pleasure for future experiences in schizophrenia spectrum disorders and major depression: A systematic review and meta-analysis. British Journal of Clinical Psychology, 58(4), 357-383. https://doi.org/ 10.1111/bjc.12218.

Hallford, D. J., Takano, K., Raes, F., \& Austin, D. W. (2019). Psychometric evaluation of an episodic future thinking variant of the autobiographical memory test-episodic future thinking-test (EFT-T). European Journal of Psychological Assessment. https://doi. org/10.1027/1015-5759/a000536

Holmes, E. A., Blackwell, S. E., Burnett Heyes, S., Renner, F., \& Raes, F. (2016). Menta imagery in depression: Phenomenology, potential mechanisms, and treatment implications. Annual Review of Clinical Psychology, 12, 249-280. https://doi.org/10. 1146/annurev-clinpsy-021815-092925.

Jing, H. G., Madore, K. P., \& Schacter, D. L. (2016, Apr). Worrying about the future: An episodic specificity induction impacts problem solving, reappraisal, and well-being. Journal of Experimental Psychology: General, 145(4), 402-418. https://doi.org/10. $1037 / x g e 0000142$.

Jing, H. G., Madore, K. P., \& Schacter, D. L. (2019, Feb). Not to worry: Episodic retrieval impacts emotion regulation in older adults. Emotion https://doi.org/10.1037/ emo0000581.

Kahneman, D., \& Tversky, A. (1981). The simulation heuristic (No. TR-5). STANFORD UNIV CA DEPT OF PSYCHOLOGY.

Lovibond, P. F., \& Lovibond, S. H. (1995, Mar). the structure of negative emotional states: Comparison of the depression anxiety stress scales (DASS) with the beck depression and anxiety inventories. Behaviour Research and Therapy, 33(3), 335-343. https://doi. org/10.1016/0005-7967(94)00075-u.

MacLeod, A. K., Tata, P., Tyrer, P., Schmidt, U., Davidson, K., \& Thompson, S. (2005, Nov). Hopelessness and positive and negative future thinking in parasuicide. British Journal of Clinical Psychology, 44(Pt 4), 495-504. https://doi.org/10.1348/ $014466505 X 35704$.

Madore, K. P., Addis, D. R., \& Schacter, D. L. (2015, Sep). Creativity and memory: Effects of an episodic-specificity induction on divergent thinking. Psychological Science, 26(9), 1461-1468. https://doi.org/10.1177/0956797615591863.

Madore, K. P., Gaesser, B., \& Schacter, D. L. (2014a). Constructive episodic simulation: Dissociable effects of a specificity induction on remembering, imagining, and describing in young and older adults. Journal of Experimental Psychology Learning Memory and Cognition, 40(3), 609-622. https://doi.org/10.1037/a0034885.

Madore, K. P., Jing, H. G., \& Schacter, D. L. (2019, 02). Selective effects of specificity inductions on episodic details: Evidence for an event construction account. Memory, 27(2), 250-260. https://doi.org/10.1080/09658211.2018.1502322.

Madore, K. P., \& Schacter, D. L. (2014b). An episodic specificity induction enhances means-end problem solving in young and older adults. Psychology and Aging, 29(4), 913-924. https://doi.org/10.1037/a0038209.

Madore, K. P., \& Schacter, D. L. (2016). Remembering the past and imagining the future: Selective effects of an episodic specificity induction on detail generation. Quarterly Journal of Experimental Psychology, 69(2), 285-298. https://doi.org/10.1080/ 17470218.2014.999097.

Newman, M. G., \& Llera, S. J. (2011). A novel theory of experiential avoidance in generalized anxiety disorder: A review and synthesis of research supporting a contrast avoidance model of worry. Clinical Psychology Review, 31, 371-382. https://doi.org/ 10.1016/j.cpr.2011.01.008.

Oettingen, G., Mayer, D., \& Portnow, S. (2016, Mar). Pleasure now, pain later: Positive fantasies about the future predict symptoms of depression. Psychological Science, 27(3), 345-353. https://doi.org/10.1177/0956797615620783.

Raes, F., Williams, J. M., \& Hermans, D. (2009, Mar). Reducing cognitive vulnerability to depression: A preliminary investigation of MEmory specificity training (MEST) in inpatients with depressive symptomatology. Journal of Behavior Therapy and Experimental Psychiatry, 40(1), 24-38. https://doi.org/10.1016/j.jbtep.2008.03.001.

Raffard, S., Esposito, F., Boulenger, J. P., \& Van der Linden, M. (2013, Oct). Impaired ability to imagine future pleasant events is associated with apathy in schizophrenia. Psychiatry Research, 209(3), 393-400. https://doi.org/10.1016/j.psychres.2013.04. 016.

Renner, F., Ji, J. L., Pictet, A., Holmes, E. A., \& Blackwell, S. E. (2017, 2017). Effects of engaging in repeated mental imagery of future positive events on behavioural activation in individuals with major depressive disorder. Cognitive Therapy and Research, 41(3), 369-380. https://doi.org/10.1007/s10608-016-9776-y.

Schacter, D. L., Addis, D. R., \& Buckner, R. L. (2007). Remembering the past to imagine the future: the prospective brain. Nature reviews neuroscience, 8(9), 657-661. https:// doi.org/10.1038/nrn2213.

Schacter, D. L., Benoit, R. G., \& Szpunar, K. K. (2017, Oct). Episodic future thinking: Mechanisms and functions. Current Opinion Science, 17, 41-50. https://doi.org/10. 1016/j.cobeha.2017.06.002.

Schubert, T., Eloo, R., Scharfen, J., \& Morina, N. (2019). How imagining personal future scenarios influences affect: Systematic review and meta-analysis. Clinical Psychology Review, 75, 101811. https://doi.org/10.1016/j.cpr.2019.101811.

Sherdell, L., Waugh, C. E., \& Gotlib, I. H. (2012, Feb). Anticipatory pleasure predicts motivation for reward in major depression. Journal of Abnormal Psychology, 121(1), 51-60. https://doi.org/10.1037/a0024945.

Skodzik, T., Adelt, M. H., Nossek, V. A., Kuck, S. T., \& Ehring, T. $(2018,10)$. Does a novel training in mental imagery reduce pathological worry? Behaviour Research and Therapy, 109, 56-67. https://doi.org/10.1016/j.brat.2018.07.005.

Skodzik, T., Leopold, A., \& Ehring, T. (2017, 01). Effects of a training in mental imagery on worry: A proof-of-principle study. Journal of Anxiety Disorders, 45, 24-33. https:/ doi.org/10.1016/j.janxdis.2016.11.009.

Snider, S. E., LaConte, S. M., \& Bickel, W. K. (2016, 07). Episodic future thinking: Expansion of the temporal window in individuals with alcohol dependence. Alcoholism: Clinical and Experimental Research, 40(7), 1558-1566. https://doi.org/10. 1111/acer.13112.

Stein, J. S., Tegge, A. N., Turner, J. K., \& Bickel, W. K. (2018, 04). Episodic future thinking reduces delay discounting and cigarette demand: An investigation of the good-subject effect. Journal of Behavioral Medicine, 41(2), 269-276. https://doi.org/10.1007/ s10865-017-9908-1.

Stöber, J. (2000). Prospective cognitions in anxiety and depression: Replication and methodological extension. Cognition \& Emotion, 14(5), 725-729. https://doi.org/10. 
1080/02699930050117693.

Szpunar, K. K., \& Schacter, D. L. (2013, May). Get real: Effects of repeated simulation and emotion on the perceived plausibility of future experiences. Journal of Experimental Psychology: General, 142(2), 323-327. https://doi.org/10.1037/a0028877.

Taylor, S. E., Pham, L. B., Rivkin, I. D., \& Armor, D. A. (1998). Harnessing the imagination: Mental simulation, self-regulation, and coping. American Psychologist, 53(4),
429-439. https://doi.org/10.1037/0003-066X.53.4.429.

Vilhauer, J. S., Cortes, J., Moali, N., Chung, S., Mirocha, J., \& Ishak, W. W. (2013, Mar). Improving quality of life for patients with major depressive disorder by increasing hope and positive expectations with future directed therapy (FDT). Innovation Clinical Neuroscience, 10(3), 12-22. 